\title{
Safety and efficacy of weekly versus three-weekly paclitaxel plus platinum neoadjuvant chemotherapy in patients with locally advanced squamous cell head and neck carcinoma: A pilot study
}

\author{
Akash Mathur, Naincy Rastogi', Dinesh Gurjar, Ramkrishna Sai,Arvind Lakesar, Hemant Malhotra
}

\begin{abstract}
Background: Squamous cell carcinoma of head and neck (SCCHN) is one of the most common cancers seen in India and also the world. Majority of patients present in locally advanced (LA) disease where neoadjuvant combination chemotherapy with a taxane plus platinum with/without 5-Fluorouracil is the standard of care treatment. There are no/few prospective trials of weekly paclitaxel in SCCHN in spite of convincing evidence regarding safety and tolerability in other solid tumors such as breast, ovary, and lung carcinoma. In the present study, we prospectively assessed the safety and efficacy of weekly versus three-weekly paclitaxel plus platinum neoadjuvant chemotherapy in patients with LA-SCCHN. Materials and Methods: We included 50 newly diagnosed patients of LA-SCCHN in the study and randomized them into two groups to receive either low-dose weekly (80 mg/sq. $\mathrm{m})$ or standard three-weekly (I75 mg/sq. m) paclitaxel along with standard dose carboplatin (AUC 5) and assessed response rates and toxicities. Results: Age and sex were evenly matched in both groups. Oral and oropharyngeal cancers were the most common sites. Hematological toxicities were significantly more in the three-weekly group. Nonhematological toxicities, especially neuropathy, were also more in this group. The overall response rate (complete response + partial response) in the three-weekly arm was $36 \%$ versus $52 \%$ in the weekly arm. Conclusion: Data from our small study suggest that weekly paclitaxel plus platinum neoadjuvant chemotherapy may be superior to the standard every 3 weeks' administration in terms of safety as well as efficacy in patients with LA-SCCHN.
\end{abstract}

Key words: Head and neck malignancies, neoadjuvant chemotherapy, paclitaxel, squamous cell carcinoma of the head and neck

\section{Introduction}

Over the past few years, India has experienced a rapid health transition with a rising burden of various cancers. Based on the cancer registry data, it is estimated that there will be about 800,000 new cancer cases in India every year. At present, cancer is the second-most common disease in India responsible for maximum mortality with about 0.3 million deaths per year. Squamous cell carcinoma of the head and neck (SCCHN) region is the most common cancer in India. In context to Jaipur city, head and neck cancers form the single largest group of cancers constituting $17.96 \%$ of all cancers with majority $(81 \%)$ of these cancers found in male patients with a M:F ratio of 4.26:1. ${ }^{[1]}$ Approximately half to three-fourths of cases of SCCHN present in locally advanced (LA) stage. Neoadjuvant chemotherapy in this subset of patients has been proven to increase progression-free survival and overall survival and is now the standard of care. Many large randomized trials have shown a survival advantage for the addition of induction chemotherapy before locoregional treatment. Two large Phase III trials demonstrated that adding taxane to platinum and 5-Fluorouracil induction chemotherapy in LA head and neck cancer significantly reduced the risk for death over that seen with PF alone. ${ }^{[2-6]}$ Furthermore, continuous low-dose paclitaxel exhibits potent antiangiogenic and proapoptotic effects in preclinical models. ${ }^{[7]}$

In the present study, we assessed in a randomized fashion, safety, and efficacy of weekly versus three-weekly paclitaxel plus platinum neoadjuvant chemotherapy in patients with LA-SCCHN.

\section{Materials and Methods}

The study population consisted of male and female patients of LA Head and Neck cancers selected from patients attending

\begin{tabular}{|l|}
\hline Access this article online \\
\hline Quick Response Code: \\
\\
Website: www.sajc.org \\
\hline DOI: 10.4103 /saj.sajc_18_17 \\
\hline
\end{tabular}

Department of Medicine, Division of Medical Oncology, SMS Medical College and Hospital, Jaipur, Rajasthan, 'Department of Pathology, Datta Meghe Institute of Medical Sciences, Wardha, Maharashtra, India Correspondence to: Dr. Hemant Malhotra, E-mail:drmalhotrahemant@gmail.com medical and R. K. Birla Cancer Centre outpatient department and medical inpatient wards. This is a hospital-based comparative study done over a period of 12 months, recruiting 50 newly diagnosed patients of LA SCCHN. Patients were randomized into two groups to receive either low-dose weekly $(80 \mathrm{mg} / \mathrm{sq} . \mathrm{m})$ or standard three-weekly $(175 \mathrm{mg} / \mathrm{sq} . \mathrm{m})$ paclitaxel along with carboplatin AUC 5. The selection of patients was done based on the standard inclusion and exclusion criteria.

Patients were randomized into two groups which were subsequently subjected to three weekly and weekly chemotherapy regimens. Three-weekly chemotherapy regime consisted of $175 \mathrm{mg} / \mathrm{m}^{2}$ of paclitaxel as a $3 \mathrm{~h}$ intravenous (IV) infusion with platinum every 3 weeks. Weekly regime consisted of $80 \mathrm{mg} / \mathrm{m}^{2}$ of paclitaxel as a $1 \mathrm{~h} \mathrm{IV}$ infusion with platinum every 3 weeks. All the patients after every 2 cycles were re-evaluated in follow-up visits. Response assessment to chemotherapy regime was evaluated every two cycles by detailed clinical examination and CT scan. Toxicities evaluated after every cycle.

\section{Statistical analysis}

Statistical analysis was done using the software SPSS version 20.0 (IBM, NY, USA). Descriptive statistics is used to describe demographic variables and clinical characteristics. Mean values along with stable disease (SD) is used for matching of cases and controls. Chi-square test is used for discrete data. Statistical significance was set at $P<0.05$.

\section{Results}

The mean age for cases in the three-weekly chemotherapy arm was $54.08 \pm 11.191$ (range 26-75 years) and in the

This is an open access journal, and articles are distributed under the terms of the Creative Commons Attribution-NonCommercial-ShareAlike 4.0 License, which allows others to remix, tweak, and build upon the work non-commercially, as long as appropriate credit is given and the new creations are licensed under the identical terms.

For reprints contact: reprints@ @medknow.com

How to cite this article: Mathur A, Rastogi N, Gurjar D, Sai R, Lakesar A, Malhotra $\mathrm{H}$. Safety and efficacy of weekly versus three-weekly paclitaxel plus platinum neoadjuvant chemotherapy in patients with locally advanced squamous cell head and neck carcinoma: A pilot study. South Asian J Cancer 2018;7:254-7. 
weekly arm was $53.88 \pm 9.536$ (range $32-70$ years). The three-weekly arm included $21(84 \%)$ males and $4(16 \%)$ females and the weekly arm had $24(96 \%)$ males and $1(4 \%)$ females. In our study, $90 \%$ of the patients were male. The M:F ratio in the three-weekly arm and weekly arm was 5.25:1 and 24:1 respectively and overall M:F ratio in the study population was 9:1. According to the primary site in the three-weekly arm, maximum reported cases were of $\mathrm{Ca}$ tongue $(20 \%)$ and in the weekly arm Ca Tonsil and Ca Buccal mucosa with $16 \%$ cases each accounted for the highest proportion of cases. Thus, the highest prevalence by site was of oral and oropharyngeal cancers. At 2 months in our study in the three-weekly arm, overall hematological toxicities were present in 12 patients $(48 \%)$ while in the weekly chemotherapy arm hematological toxicities were present in 4 patients $(16 \%)$. In the three-weekly arm, complete myelosuppression (anemia + neutropenia + thrombocytopenia) was present in 5 patients, neutropenia (Gr 3/4) in 4 patients, and thrombocytopenia ( $\mathrm{Gr} 3 / 4)$ in 3 patients as compared to 1 patient with complete myelosuppression, 2 patients of neutropenia (Gr 3/4), and 1 patient of thrombocytopenia (Gr 3/4) in the weekly arm. At 4 months in our study in the three-weekly arm, overall hematological toxicities were present in 16 patients $(64 \%)$ while in the weekly chemotherapy arm hematological toxicities were present in 8 patients $(32 \%)$. In the three-weekly arm, complete myelosuppression (anemia + neutropenia + thrombocytopenia) was present in 5 patients, neutropenia $(\mathrm{Gr} \mathrm{3/4})$ in 7 patients and thrombocytopenia (Gr 3/4) in 4 patients as compared to 3 patients with complete myelosuppression, 3 patients of neutropenia ( $\mathrm{Gr} 3 / 4)$, and 2 patients of thrombocytopenia (Gr 3/4) in the weekly arm. At 6 months in our study in the three-weekly arm, overall hematological toxicities were present in 10 patients $(40 \%)$ while in the weekly chemotherapy arm hematological toxicities were present in 2 patients $(8 \%)$. In the three-weekly arm, complete myelosuppression was

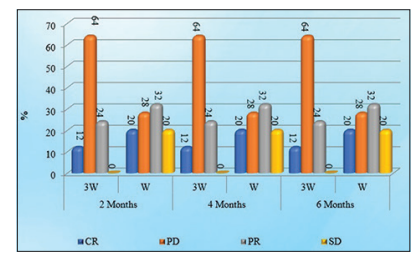

Figure 1: Distribution of the cases according to follow-up at 2, 4, and 6 months present in 1 patient, neutropenia ( $\mathrm{Gr} 3 / 4)$ in 3 patients, and thrombocytopenia ( $\mathrm{Gr} 3 / 4)$ in 6 patients as compared to no patient with complete myelosuppression, 1 patient of neutropenia ( $\mathrm{Gr} 3 / 4)$ and 1 patient of thrombocytopenia (Gr 3/4) in the weekly arm [Table 1]. At 2 months in our study in the three-weekly arm overall nonhematological toxicities were present in 6 patients $(24 \%)$ while in the weekly chemotherapy arm systemic toxicities were present in no patient $(0 \%)$. In the three-weekly arm, all the 6 patients with systemic toxicity had peripheral neuropathy. At 4 months in our study in the three-weekly arm overall nonhematological toxicities were present in 9 patients $(36 \%)$ while in the weekly chemotherapy arm systemic toxicities were present in 2 patients $(8 \%)$. In the three-weekly arm, 8 patients with systemic toxicity had peripheral neuropathy while 1 patient had deranged renal function. In the weekly arm, both patients with systemic toxicity had peripheral neuropathy. At 6 months in our study in the three-weekly arm, overall nonhematological toxicities were present in 10 patients $(40 \%)$ while in the weekly chemotherapy arm systemic toxicities were present in 4 patients (16\%) [Table 2]. In the three-weekly arm, all the 10 patients with systemic toxicity had peripheral neuropathy and in the weekly arm also all the 4 patients with systemic toxicity had peripheral neuropathy. In our study, the overall response rate in the three-weekly arm was $36 \%$ while in the weekly arm it was $52 \%$ at 2,4 , and 6 months. In the three-weekly arm, $36 \%$ of patients showed response $(12 \%$ complete response [CR] and $24 \%$ partial response [PR]), $64 \%$ patients had progressive disease (PD) while none of the patients had SD. In the weekly arm, $52 \%$ of patients showed a response ( $20 \% \mathrm{CR}$ and $32 \% \mathrm{PR}), 28 \%$ patients had PD while $20 \%$ of the patients had SD [Figure 1]. Noncompliance to locoregional treatment after neoadjuvant chemotherapy was seen in $28 \%$ patients in the three-weekly arm and $20 \%$ patients in the weekly arm. Overall noncompliance for curative treatment was seen in $48 \%$ patients in our study postneoadjuvant chemotherapy.

\section{Discussion}

Sharma et al., in their review titled "Spectrum of malignancies in Jaipur region (2004-2008)" observed that head and neck cancers formed the single largest group in their study $(17.96 \%$ of all cancers) with majority (81\%) of these cancers found in male patients with M:F ratio of $4.26: 1 .^{[1]}$ Similar results in our study

Table 1: Distribution of the cases according to hematological toxicities at 2, 4, and 6 months

\begin{tabular}{|c|c|c|c|c|c|c|c|c|c|c|c|c|}
\hline \multirow[t]{3}{*}{ Haematological toxicities } & \multicolumn{2}{|c|}{$3 \mathrm{~W}$} & \multicolumn{2}{|c|}{ W } & \multicolumn{2}{|c|}{$3 \mathrm{~W}$} & \multicolumn{2}{|c|}{ W } & \multicolumn{2}{|c|}{$3 \mathrm{~W}$} & \multicolumn{2}{|c|}{ W } \\
\hline & No. & $\%$ & No. & $\%$ & No. & $\%$ & No. & $\%$ & No. & $\%$ & No. & $\%$ \\
\hline & \multicolumn{4}{|c|}{2 Months } & \multicolumn{4}{|c|}{4 Months } & \multicolumn{4}{|c|}{6 Months } \\
\hline Absent & 13 & 52 & 21 & 84 & 9 & 36 & 17 & 68 & 15 & 60 & 23 & 92 \\
\hline Present & 12 & 48 & 4 & 16 & 16 & 64 & 8 & 32 & 10 & 40 & 2 & 8 \\
\hline Chi-square & \multicolumn{4}{|c|}{4.504 with $1 \mathrm{df} ; P=0.034$} & \multicolumn{4}{|c|}{3.926 with $1 \mathrm{df} ; P=0.048$} & \multicolumn{4}{|c|}{5.373 with $1 \mathrm{df} ; P=0.020$} \\
\hline
\end{tabular}

Table 2: Distribution of the cases according to nonhematological toxicities at 2, 4, and 6 months

\begin{tabular}{|c|c|c|c|c|c|c|c|c|c|c|c|c|}
\hline \multirow[t]{3}{*}{ Non-haematological toxicities } & \multicolumn{2}{|c|}{$3 \mathbf{W}$} & \multicolumn{2}{|c|}{$\mathbf{W}$} & \multicolumn{2}{|c|}{$3 \mathrm{~W}$} & \multicolumn{2}{|c|}{ W } & \multicolumn{2}{|c|}{$3 \mathbf{W}$} & \multicolumn{2}{|c|}{$\mathbf{W}$} \\
\hline & No. & $\%$ & No. & $\%$ & No. & $\%$ & No. & $\%$ & No. & $\%$ & No. & $\%$ \\
\hline & \multicolumn{4}{|c|}{2 months } & \multicolumn{4}{|c|}{ 4months } & \multicolumn{4}{|c|}{6 months } \\
\hline Absent & 19 & 76 & 25 & 100 & 16 & 64 & 23 & 92 & 15 & 60 & 21 & 84 \\
\hline Present & 6 & 24 & 0 & 0 & 9 & 36 & 2 & 8 & 10 & 40 & 4 & 16 \\
\hline Chi-square & \multicolumn{4}{|c|}{4.735 with $1 \mathrm{df} ; P=0.030$} & \multicolumn{4}{|c|}{4.196 with $1 \mathrm{df} ; P=0.041 \mathrm{~S}$} & \multicolumn{4}{|c|}{5.373 with $1 \mathrm{df} ; P=0.020 \mathrm{~S}$} \\
\hline
\end{tabular}


suggest that this high frequency of these cancers among male population is probably because of the increased use of tobacco products. The highest prevalence by site of oral and oropharyngeal cancers can again be linked to increased use of tobacco products.

In 2013, Shimizu et al., in a comparative analysis of carboplatin and paclitaxel combination chemotherapy schedules (weekly vs. three weekly) in previously untreated patients with advanced non-small cell lung cancer found hematological toxicities to be $48 \%$ and $83 \% \cdot{ }^{[4]}$ Hence, while we found less hematological side effects in both the arms as compared to the above study, weekly arm had significantly better side effect profile similar to the above mentioned study with the difference of hematological toxicities in the two arms being statistically significant at 2 months $(P=0.034)$, 4 months $(P=0.048)$, and 6 months $(P=0.020)$. In 2013 Glaze et al. while evaluating dose-dense (weekly) paclitaxel with carboplatin for advanced ovarian cancer found 59\% hematological toxicities, ${ }^{[8]}$ El-Shenshawy et al., in 2012 in a study to evaluate the efficacy and toxicity of dose-dense weekly paclitaxel plus 3 weeks carboplatin as a neoadjuvant and adjuvant therapy for primary epithelial ovarian cancer, ${ }^{\left[{ }^{[9}\right.}$ The Multicentre Italian Trials in Ovarian Cancer- $7^{[10]}$ reiterated the observations made in our study finding significantly less hematological toxicities with weekly chemotherapy regimen.

Furthermore, there was statistically significant difference in nonhematological toxicities seen in the two arms at 2 months $(P=0.030), 4$ months $(P=0.041)$, and 6 months $(P=0.020)$. Belani et al. in NSCLC also observed an increased incidence of peripheral neuropathy in the three-weekly arm (18\% in three weekly vs. $12 \%$ in weekly). ${ }^{[1]}$ In our study also, we obtained similar results. El-Shenshawy et al. in epithelial ovarian cancers observed that the incidence of peripheral neuropathy was $10 \% \cdot{ }^{[9]}$ These results were comparable to those obtained in our study. Slightly higher incidence of peripheral neuropathy at 6 months' follow-up with weekly chemotherapy might be due to smaller sample size or due to confounding factors like smoking which are also independent risk factors for peripheral neuropathy. In 2004, Polee et al. in Phase I pharmacokinetic study of weekly paclitaxel and carboplatin in patients with metastatic esophageal cancer $^{[6]}$ made observations similar to our study with significantly better neurotoxicity profile in the weekly arm. In 2009, Mohamad A. Hassan in his study titled "Carboplatin and Weekly Paclitaxel in Metastatic and LA Breast Cancer Patients" and Ignace Vergote et al. in 2015 in Phase II study of weekly paclitaxel/carboplatin in the treatment of gynecologic cancers also made similar observations.

In 2008, Belani et al., in a randomized Phase III study of weekly paclitaxel in combination with carboplatin versus standard every 3 weeks' administration of carboplatin and paclitaxel for patients with previously untreated advanced non-small cell lung cancer observed that the response rate was $27.6 \%$ for weekly arm and $19.2 \%$ for three-weekly arm. ${ }^{[11]}$ In our study, we found a significant difference in the response rates with $52 \%$ for weekly versus $36 \%$ for three-weekly arm. Green et al. in their study to determine the impact of change in schedule of paclitaxel administration from once every 3 weeks to frequent administration on the CR rate in the breast and lymph nodes for patients with invasive breast cancer treated with primary systemic chemotherapy similar to our study concluded that the change in schedule of paclitaxel from once every 3 weeks to a more frequent administration significantly improved the ability to eradicate invasive cancer in the breast and lymph nodes. ${ }^{[3]}$ In 2004, Polee et al. similar to our study found weekly chemotherapy regimen to be tolerable and effective in Metastatic Esophageal Cancer. ${ }^{[6]}$ In 2002 D'Addario et al. assessed the feasibility and toxicity of weekly paclitaxel-carboplatin in 131 patients with pretreated and non-pretreated solid tumors and made similar observations. ${ }^{[12]}$ The response rate is high including CRs and responses appear quickly, making this therapy most suitable for multimodal and neoadjuvant treatments.

In our study, noncompliance to curative treatment after neoadjuvant chemotherapy was found in $28 \%$ patients in the three-weekly arm and $20 \%$ patients in the weekly arm. Overall noncompliance for curative treatment was seen in $48 \%$ patients post neoadjuvant chemotherapy. Mohanti et al. in their study observed that the rate of noncompliance for curative treatment in head and neck cancers reported at a tertiary care center in New Delhi was as high as $38 \% .{ }^{[13]}$ In our study, we observed a slightly higher noncompliance rate of $48 \%$. This difference can be attributed to either a smaller sample size or other independent confounding factors like literacy rate which is lower in Rajasthan as compared to New Delhi.

In our study, we found that in patients who are matched with regards to age, sex, stage of disease, and performance status the hematological and systemic toxicities are significantly lower in the weekly chemotherapy arm. It was also observed that the patients receiving weekly chemotherapy have a better outcome in terms of response rates.

To the best of our knowledge, this study is the first study done to evaluate the safety and efficacy of weekly versus three weekly paclitaxel plus platinum neoadjuvant chemotherapy in patients with LA head and neck squamous cell carcinoma in Indian population.

\section{Conclusion}

Our relatively small study suggests that weekly paclitaxel plus platinum neoadjuvant chemotherapy could be superior to the standard 3 weeks administration of this agent both in terms of safety (adverse effect profile) and efficacy (response rates) in patients with LA head and neck squamous cell carcinoma. Higher noncompliance rates for curative treatment in head and neck cancer patients is a serious issue that can compromise treatment, lead to suboptimal outcomes and hence warrants urgent interventions in the form of proper counseling and follow-up of all patients. Although there is available published literature signifying that weekly paclitaxel plus platinum is superior in terms of safety and efficacy in breast, ovary and lung carcinoma, our study comparing weekly versus three-weekly dosing of paclitaxel in the setting of head and neck malignancies, which is one of the most prevalent cancers in India, may be a significant advance in the treatment of this group of cancers.

Financial support and sponsorship

Nil.

\section{Conflicts of interest}

There are no conflicts of interest. 


\section{References}

1. Sharma RG, Kapoor R, Bang BA, Gurjar K. Spectrum of malignancies in Jaipur region (2004-2008). Indian J Cancer 2014;51:45-53.

2. Vokes EE. Induction chemotherapy for head and neck cancer: Recent data. Oncologist 2010;15 Suppl 3:3-7.

3. Green MC, Buzdar AU, Smith T, Ibrahim NK, Valero V, Rosales MF, et al. Weekly paclitaxel improves pathologic complete remission in operable breast cancer when compared with paclitaxel once every 3 weeks. J Clin Oncol 2005;23:5983-92.

4. Shimizu T, Yokoi T, Tamaki T, Kibata K, Inagaki N, Nomura S, et al. Comparative analysis of carboplatin and paclitaxel combination chemotherapy schedules in previously untreated patients with advanced non-small cell lung cancer. Oncol Lett 2013;5:761-7.

5. Harano K, Terauchi F, Katsumata N, Takahashi F, Yasuda M, Takakura S, et al. Quality-of-life outcomes from a randomized phase III trial of dose-dense weekly paclitaxel and carboplatin compared with conventional paclitaxel and carboplatin as a first-line treatment for stage II-IV ovarian cancer: Japanese Gynecologic Oncology Group Trial (JGOG3016). Ann Oncol 2014;25:251-7.

6. Polee MB, Sparreboom A, Eskens FA, Hoekstra R, van de Schaaf J, Verweij J, et al. A phase I and pharmacokinetic study of weekly paclitaxel and carboplatin in patients with metastatic esophageal cancer. Clin Cancer Res 2004; 10: 1928-34.

7. Symmans FW. Breast cancer response to paclitaxel in vivo. Drug Resist
Updat 2001;4:297-302.

8. Glaze S, Teitelbaum L, Chu P, Ghatage P, Nation J, Nelson G, et al. Dose-dense paclitaxel with carboplatin for advanced ovarian cancer: A feasible treatment alternative. J Obstet Gynaecol Can 2013;35:61-7.

9. El-Shenshawy H, Taema S, El-Zahaf E, El-Beshbeshi W, Sharaf Eldeen D, Fathy A. Advanced non-small cell lung cancer in elderly patients: The standard every 3-weeks versus weekly paclitaxel with carboplatin. Egypt J Chest Dis Tuberc 2012;61:485-93.

10. Pignata S, Scambia G, Katsaros D, Gallo C, Pujade-Lauraine E, De Placido S, et al. Carboplatin plus paclitaxel once a week versus every 3 weeks in patients with advanced ovarian cancer (MITO-7): A randomised, multicentre, open-label, phase 3 trial. Lancet Oncol 2014; 15:396-405.

11. Belani CP, Ramalingam S, Perry MC, LaRocca RV, Rinaldi D, Gable PS, et al. Randomized, phase III study of weekly paclitaxel in combination with carboplatin versus standard every-3-weeks administration of carboplatin and paclitaxel for patients with previously untreated advanced non-small-cell lung cancer. J Clin Oncol 2008;26:468-73.

12. D'Addario G, Morant R, Boehme C, Cerny T. Feasibility and toxicity of weekly paclitaxel-carboplatin in 131 patients with pretreated and non-pretreated solid tumors. Onkologie 2002;25:152-7.

13. Mohanti BK, Nachiappan P, Pandey RM, Sharma A, Bahadur S, Thakar A, et al. Analysis of 2167 head and neck cancer patients' management, treatment compliance and outcomes from a regional cancer centre, Delhi, India. J Laryngol Otol 2007;121:49-56. 